\title{
Influence of loading pattern in fatigue life for notched round bars subjected to bending-torsion
}

\author{
Ricardo Branco ${ }^{1,2, a}$, J.D. Costa ${ }^{2}$ and F.V. Antunes ${ }^{2}$ \\ ${ }^{1}$ Department of Mechanical Engineering, ISEC, Polytechnic Institute of Coimbra, Quinta da Nora, \\ 3030-199 Coimbra, Portugal \\ ${ }^{2}$ CEMUC, Department of Mechanical Engineering, University of Coimbra, Rua Luís Reis Santos, \\ Pinhal de Marrocos, 3030-788 Coimbra, Portugal
}

\begin{abstract}
Fatigue behaviour of DIN 34CrNiMo6 lateral notched round bars subjected to in-phase bending-torsion loading was investigated. Experimental tests were conducted under constant amplitude loading with stress ratios close to zero. Crack initiation and crack growth were monitored in-situ using a high-resolution digital system. Fracture surfaces were examined by scanning electron microscope (SEM). A multi-crack initiation phenomenon from material defects at the notch surface was found. The SEM analyses revealed the presence of relatively high inclusions with sizes ranging from 2 to $30 \mu \mathrm{m}$. Finally, fatigue life predictions were carried out using both the Coffin-Manson (CM) and the Smith-WatsonTopper (SWT) models. The notch effect was simulated with the Theory of Critical Distances (TCD) and the Equivalent Strain Energy Density (ESED) concept. Regardless of the model, very good correlations between experimental and predicted fatigue lives were observed, particularly for lives greater than $10^{4}$ cycles.
\end{abstract}

\section{Introduction}

DIN 34CrNiMo6 high strength steel is a very versatile engineering material. It combines high ductility, deep hardenability, toughness and strength. Due to these exceptional properties, it is ideal for critical components such as pinions, crankshafts, connecting rods, axles, torsion bars, among others. These components have severe geometric discontinuities and work under complex loading. The presence of localised high stress concentration effects and multiaxial loading make them susceptible to fatigue failure.

In this research, fatigue behaviour of lateral notched round bars made of DIN 34CrNiMo6 high strength steel subjected to in-phase combined bending-torsion loading is investigated. Tests were conducted under constant-amplitude loading with stress ratios close to zero.



This is an Open Access article distributed under the terms of the Creative Commons Attribution License 4.0, which permits unrestricted use, distribution, and reproduction in any medium, provided the original work is properly cited. 

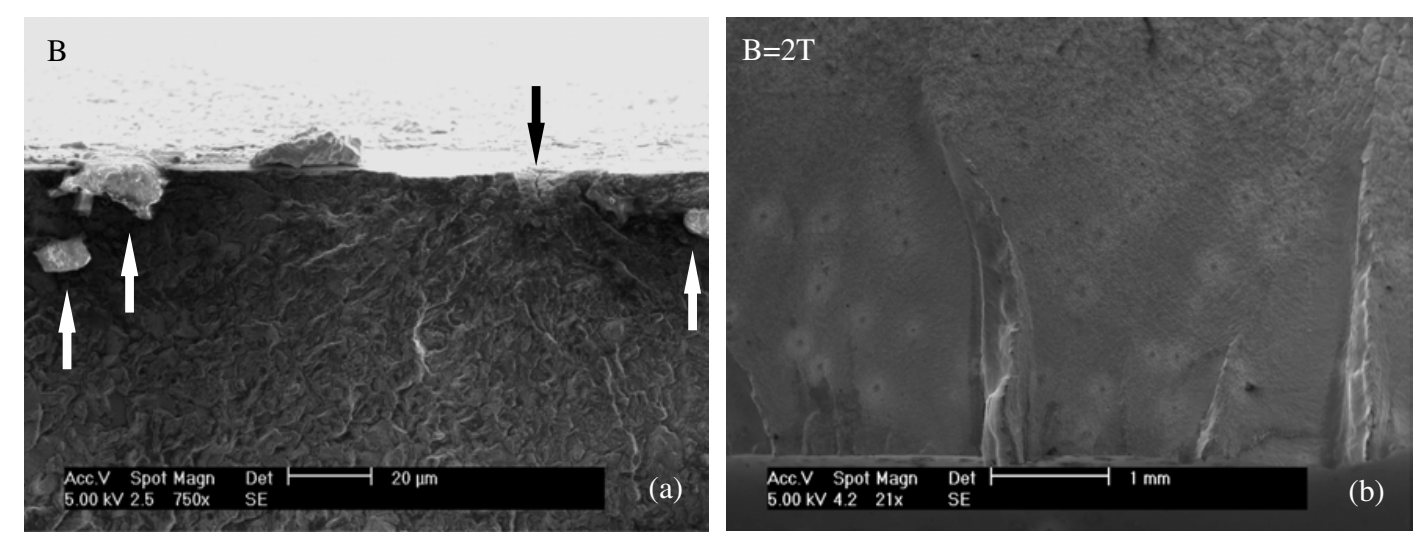

Figure 1. SEM micrographs of fracture surfaces.

\section{Procedure}

The material used was a DIN 34CrNiMo6 high strength steel. Two lateral U-shaped notched specimens were used (16-mm diameter solid round bar with a $3 \mathrm{~mm}$-diameter and $3 \mathrm{~mm}$-depth notch and a 14-mm diameter solid round bar with a $3 \mathrm{~mm}$-diameter and $3 \mathrm{~mm}$-depth notch). Fatigue tests were performed under constant-amplitude loading with stress ratios close to zero $(\mathrm{R} \approx 0)$. The loading scenarios studied were single bending, single torsion and bending-torsion loading. Regarding the last type, three different ratios of the bending moment $(\mathrm{B})$ to the torsion moment $(\mathrm{T})$ were adopted, more specifically $\mathrm{B}=2 \mathrm{~T}$, $\mathrm{B}=\mathrm{T}$ and $\mathrm{B}=2 \mathrm{~T} / 3$.

\section{Results}

The SEM analysis of the fracture surfaces evidenced multi-crack initiation phenomena from pores and inclusions at the notch surface. Figure 1a exhibits a representative case of crack initiation occurred from an inclusion (black arrow). The direction of propagation is from the top to the bottom. The white arrows show more inclusions near the surface of the notch. In this material, relatively high inclusions with sizes ranging from 2 to $30 \mu \mathrm{m}$ were found. Figure $1 \mathrm{~b}$ shows several steps at the fracture surfaces which can be associated with the junction of different propagation planes caused by nucleation of various cracks at the periphery of the notch surface.

A significant influence of the loading conditions on the surface crack trajectories, initiation sites and surface crack angles at the early stage of crack growth was observed. These variables were successfully predicted using criteria based on the principal stress field. Under single bending, the crack propagates in a direction approximately normal to the axis of the specimen. In the other cases, the crack trajectories become more and more curved due to an increase of the shear stress to the normal stress ratio.

Fatigue life predictions were carried out using both the CM and the SWT models. The notch effect was simulated with the Theory of Critical Distances and the Equivalent Strain Energy Density concept. A finite element model was developed to evaluate the stress-strain state at the notch region. The fatigue damage parameter was the local von Mises equivalent stress. This parameter revealed adequate to correlate resultant stress-strain states with the fatigue life. Regardless of the model, very good correlations between experimental and predicted fatigue lives were observed, particularly for lives greater than $10^{4}$ cycles. 


\section{Conclusions}

In this research, fatigue behaviour of lateral notched round bars made of DIN 34CrNiMo6 high strength steel subjected to combined bending-torsion loading was investigated. The fracture surfaces revealed a multi-crack initiation phenomenon. Initiation process occurred form inclusions and pores located at the notch surface. The fatigue life prediction obtained from both the CM and SWT models led to accurate results. 